\title{
Relaciones Anatómicas y Correlaciones Estadísticas entre los Ligamentos Meniscofemorales y el Ligamento Cruzado Posterior en Individuos Chilenos
}

\author{
Anatomical Relationships and Statistical Correlations between the Meniscofemoral \\ Ligaments and the Posterior Cruciate Ligament in Chilean Individuals
}

Villarroel, G.* \& Olave, E.**

VILLARROEL, G. \& OLAVE, E. Relaciones anatómicas y correlaciones estadísticas entre los ligamentos meniscofemorales y el ligamento cruzado posterior en individuos chilenos. Int. J. Morphol., 34(4):1259-1265, 2016.

RESUMEN: Entre las estructuras que conforman la articulación de la rodilla, los ligamentos meniscofemorales (LMF) son considerados como elementos inconstantes, que están en relación al ligamento cruzado posterior (LCP). Con el propósito de correlacionar los LMF con el LCP y clasificar al ligamento meniscofemoral posterior (LMFP) con respecto al LCP, se estudiaron 30 rodillas de individuos Chilenos, fijadas en formaldehído al $10 \%$ y pertenecientes a los Laboratorios de Anatomía, de la Facultad de Medicina de la Universidad Católica del Maule. Las medidas fueron registradas con un cáliper electrónico y se fotografiaron las muestras. Con respecto al trayecto de las fibras del LMFP, se observó un trayecto recto en $76 \%$ los casos (tipo I) y sus fibras rotando sobre su eje en el porcentaje restante (tipo II). Además las fibras del LMFP se fundieron con las del LCP (66,7 \%) y en menor porcentaje van directa e independientemente al cóndilo femoral medial. Se obtuvieron los promedios y se correlacionaron las variables a estudiar. De esta manera se determinó que existe correlación entre la distancia epicóndilo lateral del fémur- maleólo lateral (ELF-ML) y la longitud del LCP, y de esta misma distancia con la longitud del LMFP. Además hay correlación entre la longitud del LCP y la longitud del LMFP.

PALABRAS CLAVE: Anatomía; Rodilla; Ligamento meniscofemoral anterior; Ligamento meniscofemoral posterior; Ligamento cruzado posterior.

\section{INTRODUCCIÓN}

La anatomía de la articulación de la rodilla tiene interés debido a su importancia en las actividades de marcha, carrera y salto, además de su función estática en la posición de bipedestación. Dentro de sus componentes existen dos ligamentos cruzados que se extienden desde las áreas intercondíleas de la tibia hasta los cóndilos femorales. El ligamento cruzado posterior (LCP) se origina del área intercondílea posterior de la tibia en dirección ascendente, anterior y medial, terminando en una línea de inserción horizontal en la parte anterior de la cara intercondílea del cóndilo medial del fémur y en el fondo de la fosa intercondílea (Rouvière \& Delmas, 2005). El LCP está reforzado por dos ligamentos que unen el asta posterior del menisco lateral a la cara lateral del cóndilo medial del fémur en la incisura intercondílea, denominándose a estas estructuras ligamen- tos meniscofemorales (LMF). El ligamento meniscofemoral anterior (LMFA) se ubica ventral al LCP, conociéndose a éste en la literatura médica como ligamento de Humphrey. El ligamento meniscofemoral posterior (LMFP) transcurre dorsal al LCP y se conoce como ligamento de Wrisberg (Brantigan \& Voshell, 1946).

Gupte et al. (2003) realizaron una revisión de la literatura y concluyeron que de un total 1022 rodillas estudiadas, un $91 \%$ presentó al menos uno de los ligamentos mencionados. La mayoría de los autores reportaron mayor prevalencia del LMFP (Radoïévitch, 1931; Brantigan \& Voshell; Candiolo \& Gautero, 1959; Kusayama et al., 1994; Harner et al., 1995; Wan \& Felle, 1995; Poynton et al., 1997; Cho et al., 1999).

\footnotetext{
* Departamento de Salud, Universidad de Los Lagos, Osorno, Chile.

** Facultad de Medicina, Universidad de La Frontera, Temuco, Chile.
} 
Debido a la relación de estos ligamentos con el LCP y que en la revisión bibliográfica se encontró escasa información, el propósito del presente trabajo es correlacionar los LMF con el LCP. Con los datos obtenidos se espera contribuir con un soporte morfológico a la clínica quirúrgica de estas importantes estructuras de la rodilla.

\section{MATERIAL Y MÉTODO}

Se disecaron 30 miembros inferiores (16 derechos y 14 izquierdos), de individuos chilenos de sexo no determinado. Estas piezas se encontraban fijadas en una solución de formaldehído al $10 \%$ y todas pertenecientes al Departamento de Anatomía de la Universidad Católica del Maule, Talca, Chile.

Para disección mesoscópica se utilizó material quirúrgico y una lupa marca Ransor $^{\circledR}(5 \mathrm{X})$. La delimitación y disección de la región de la rodilla se prosiguió según lo descrito por Testut \& Jacob (1961).

Se obtuvieron las siguientes medidas:

- Distancia epicóndilo lateral del fémur-maléolo lateral (ELFML).

- Longitud de los ligamentos.

- Ancho medio de los ligamentos.

Las medidas se expresaron en milímetros $(\mathrm{mm})$ utilizando un cáliper electrónico digital, marca Mitutoyo ${ }^{\circledR}$ de precisión $0,01 \mathrm{~mm}$. Una vez realizada la disección y registradas las medidas, las muestras fueron fotografiadas. Se realizó un análisis estadístico descriptivo y un análisis de correlación con el programa GraphPad Prism versión 6,01.

\section{RESULTADOS}

El LMFA estuvo presente en 13 rodillas $(43,4 \%)$ y el LMFP en 21 rodillas (70\%). Las correlaciones se realizaron a través de la prueba de Pearson, utilizándose los parámetros promedios mostrados en la Tabla I.

Las correlaciones efectuadas fueron las siguientes:

a) Correlación entre la distancia ELF-ML y longitud LCP. Se determinó correlación positiva moderada entre ambos parámetros de $\mathrm{r}=0,43$ y con un $\mathrm{r}^{2}=0,18$. Con un valor $\mathrm{p}$ de 0,02 (estadísticamente significativo) y un intervalo de confianza (95\%) que va desde 0,09 a 0,69 (Fig. 1).
Tabla I. Parámetros biométricos de los ligamentos LMF, LCP y distancia ELF-ML.

\begin{tabular}{lr}
\hline Parámetro & \multicolumn{1}{c}{ Promedio } \\
\hline Distancia ELF -ML & $393,62 \mathrm{~mm}$ \\
Longitud del LCP & $41,52 \mathrm{~mm}$ \\
Ancho medio del LCP & $10,92 \mathrm{~mm}$ \\
Longitud del LMFA & $29,04 \mathrm{~mm}$ \\
Ancho LMFA & $4,95 \mathrm{~mm}$ \\
Longitud del LMFP & $27,46 \mathrm{~mm}$ \\
Ancho medio del LMFP & $4,06 \mathrm{~mm}$ \\
\hline
\end{tabular}

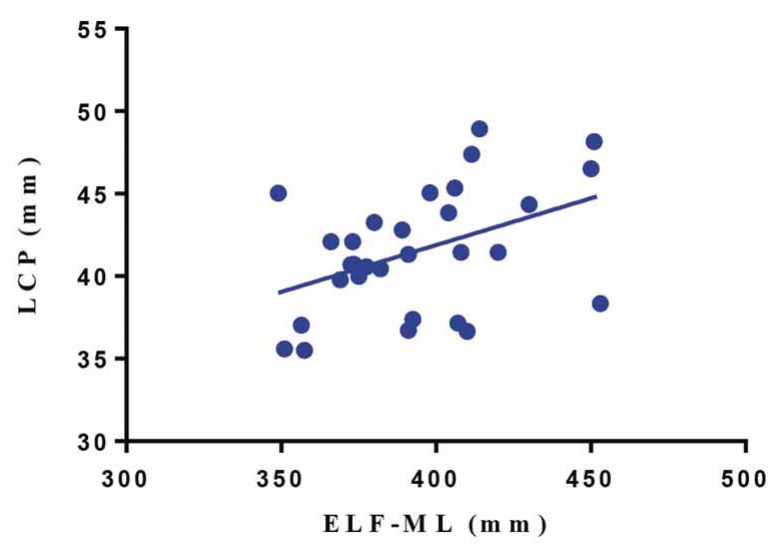

Fig. 1. Diagrama de dispersión donde se observa la relación existente entre la variable longitud del LCP y la distancia ELF-ML.

b) Correlación entre la distancia ELF-ML y longitud LMFA. No existe relación entre ambas variables, con un $\mathrm{r}=$ 0,07 y un valor $\mathrm{p}=0,81$ no estadísticamente significativo (Fig. 2).

c) Correlación entre la distancia ELF-ML y longitud LMFP. El coeficiente de correlación ( $r=0,61)$ determinó que ésta es positiva moderada entre ambas variables, con un $\mathrm{r} 2=0,37$, un valor $\mathrm{p}=0,003$ (estadísticamente significativo) y un intervalo de confianza ( $95 \%$ ) que va desde 0,24 a 0,82 (Fig. 3).

d) Correlación entre la longitud del LCP y la longitud del LMFA. Se observó correlación positiva baja entre las longitudes de ambos ligamentos con un $\mathrm{r}=0,25 \mathrm{y}$ un valor $\mathrm{p}=0,42$ (no estadísticamente significativo; Fig. 4).

e) Correlación entre la longitud del LCP y la longitud del LMFP. Se determinó correlación positiva moderada entre ambos parámetros de $\mathrm{r}=0,47$, con un $\mathrm{r}^{2}=22$, un valor de $\mathrm{p}=0,03$ (estadísticamente significativo) y un intervalo de confianza (95\%) de 0,04 a 0,75 (Fig. 5). 


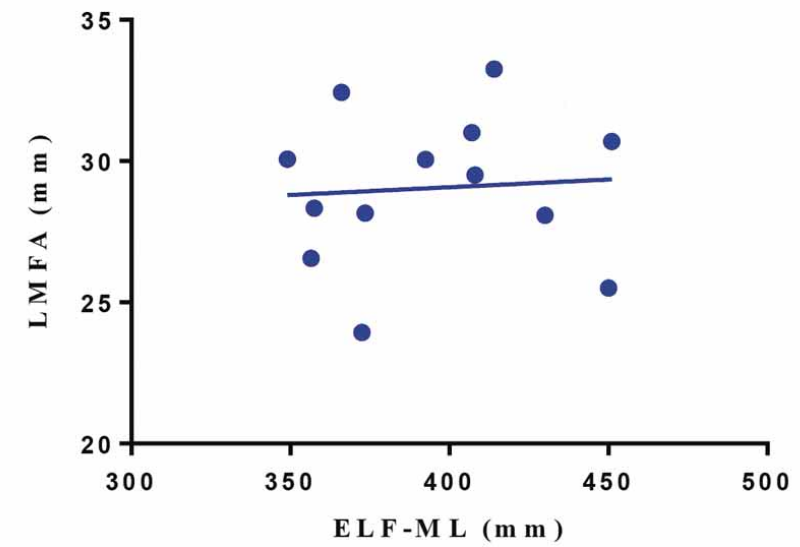

Fig. 2. Diagrama de dispersión que representa la relación entre las variable longitud del LMFA y la distancia ELF-ML.

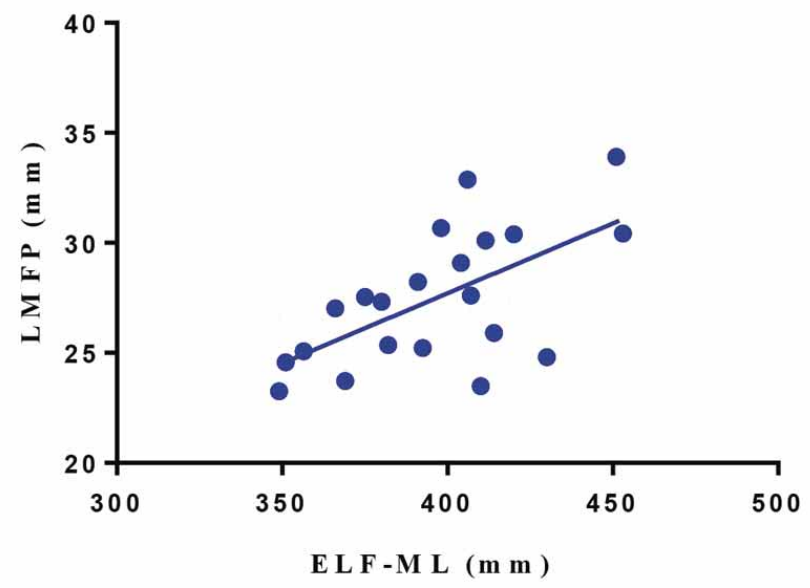

Fig. 3. Diagrama de dispersión que representa la relación entre la longitud del LMFP y la distancia ELF-ML.

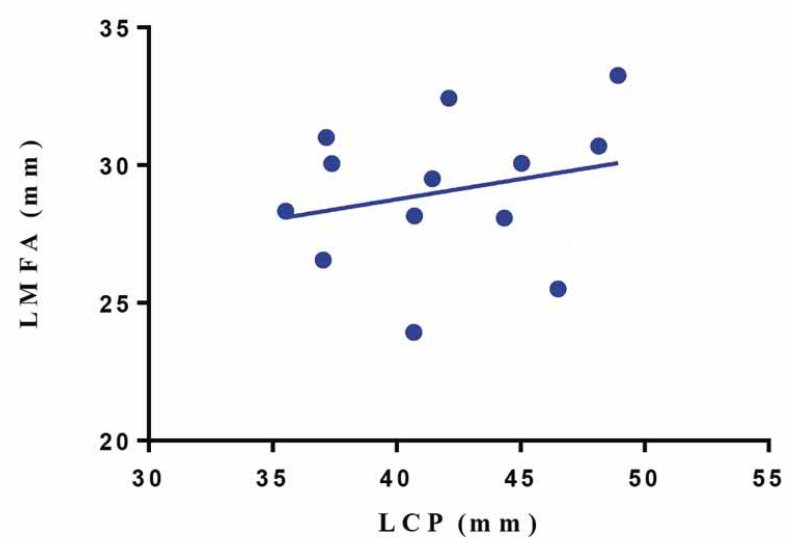

Fig. 4. Diagrama de dispersión que representa la relación entre las longitudes del LCP y el LMFA. f) Correlación entre el ancho medio del LCP con el ancho medio del LMFA. Se determinó una correlación negativa baja con un $r=-0,20$ y valor $\mathrm{p}=0,51$ (no estadísticamente significativo; Fig. 6).

g) Correlación entre el ancho medio del LCP con el ancho medio del LMFP. No existe correlación entre estos dos parámetros con un $\mathrm{r}=0,05$ y un valor $\mathrm{p}=0,81$ (no estadísticamente significativo; Fig. 7).

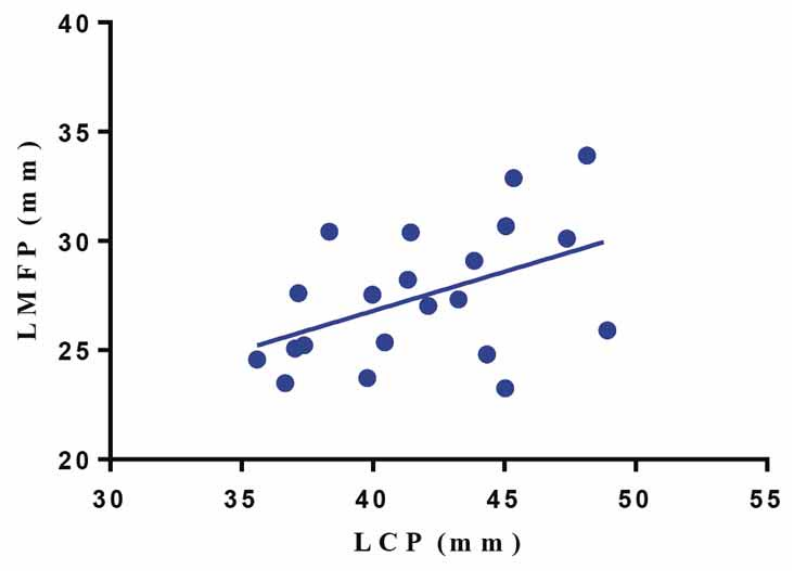

Fig. 5. Diagrama de dispersión que muestra la relación entre las longitudes del LCP y el LMFP.

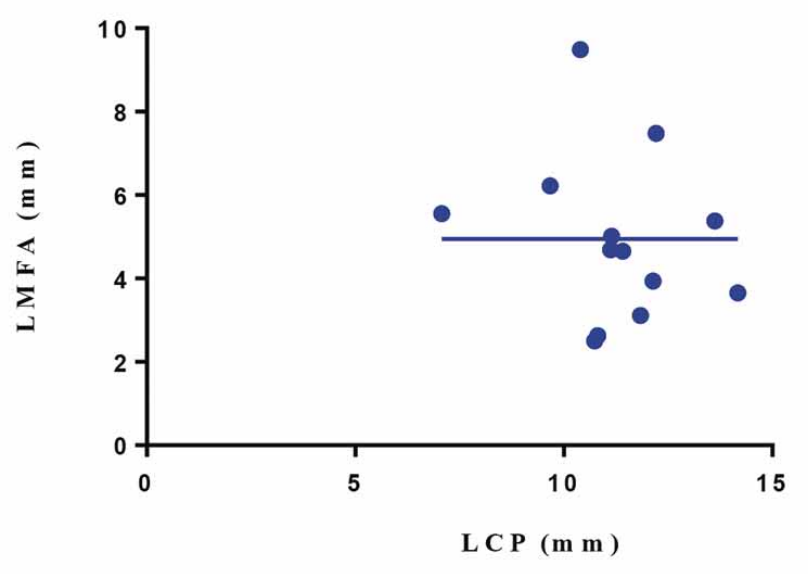

Fig. 6. Diagrama de dispersión que muestra la relación entre los anchos del LCP y el LMFA.

h) Se realizó una clasificación para los LMFP. Dividiéndolos en 2 tipos; el tipo I (Fig. 8-9-12) presenta una trayectoria recta y el tipo II (Fig. 10-11) rota en su trayecto. Se realiza una sub clasificación en relación a la adherencia del ligamento meniscofemoral posterior al ligamento cruzado posterior (Tabla II): 
A: fibras del LMFP se unen con el LCP cuando entra en contacto con éste (Fig. 8)

B: fibras del LMFP se unen en el tercio final de su trayectoria al LCP (Fig. 9).

C: no se unen fibras del LMFP con el LCP (Fig.12).

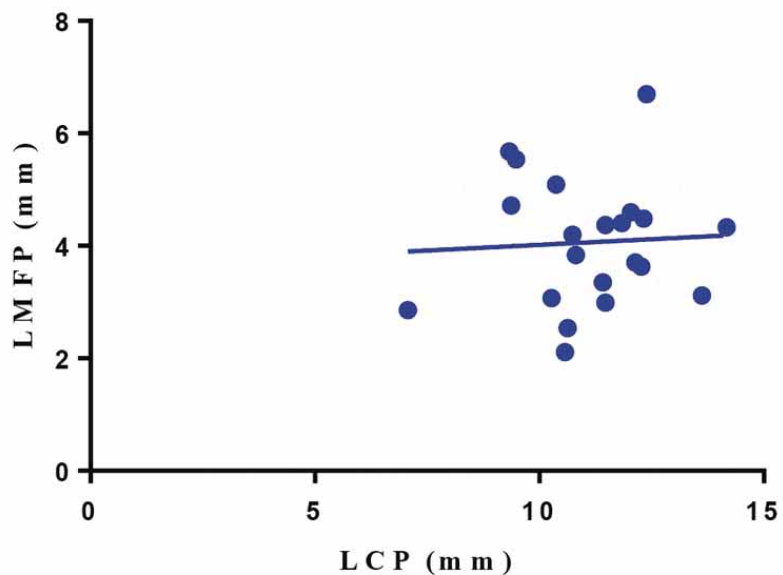

Fig. 7. Diagrama de dispersión que representa la relación entre los anchos del LCP y el LMFP.

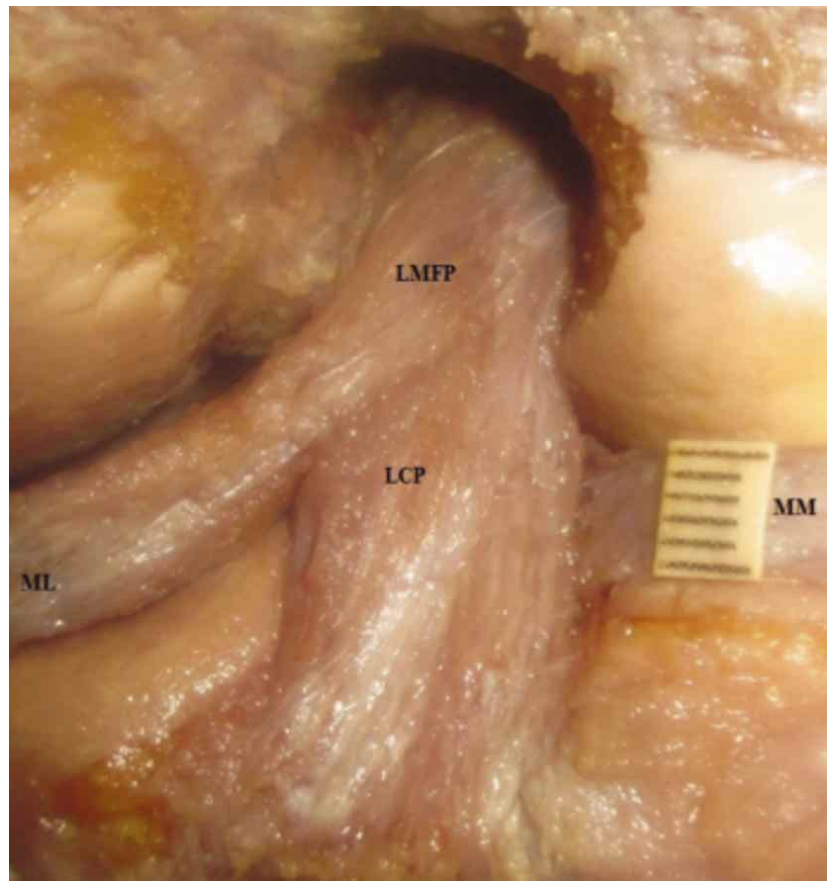

Fig. 8. Vista posterior de la articulación de rodilla izquierda. LMFP. Ligamento meniscofemoral posterior; LCP. Ligamento cruzado posterior; MM. Menisco medial; ML. Menisco lateral. El LMFP muestra una trayectoria tipo I relación tipo A.

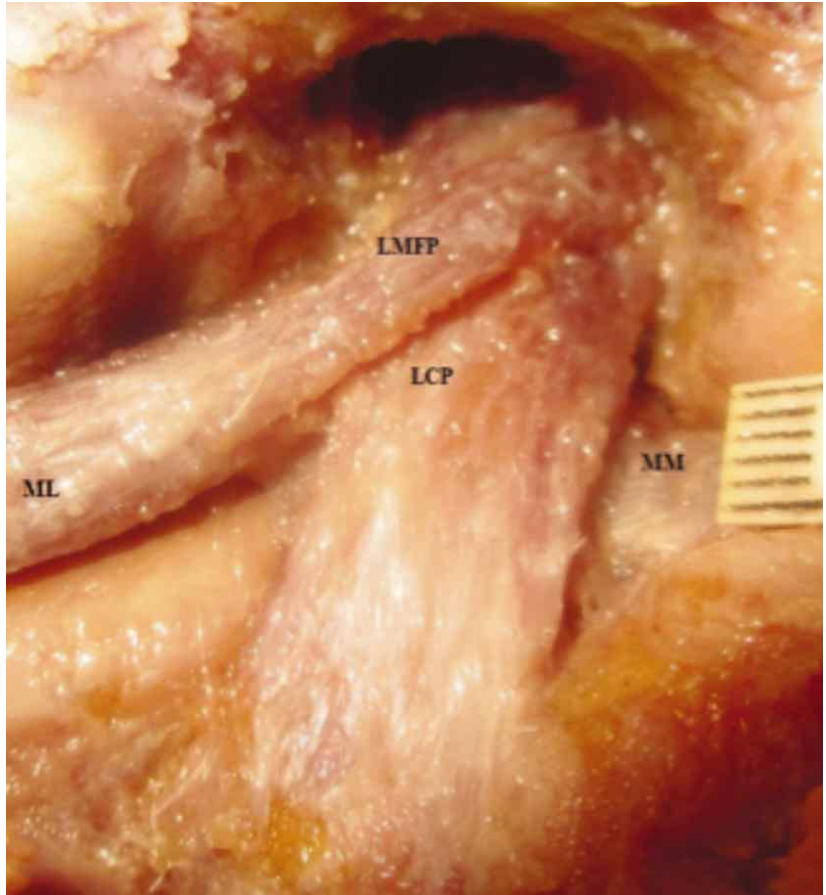

Fig. 9. Vista posterior de la articulación de la rodilla izquierda. LMFP. Ligamento meniscofemoral posterior; LCP. Ligamento cruzado posterior; MM. Menisco medial; ML. Menisco lateral. El LMFP presenta una trayectoria tipo I relación tipo B.

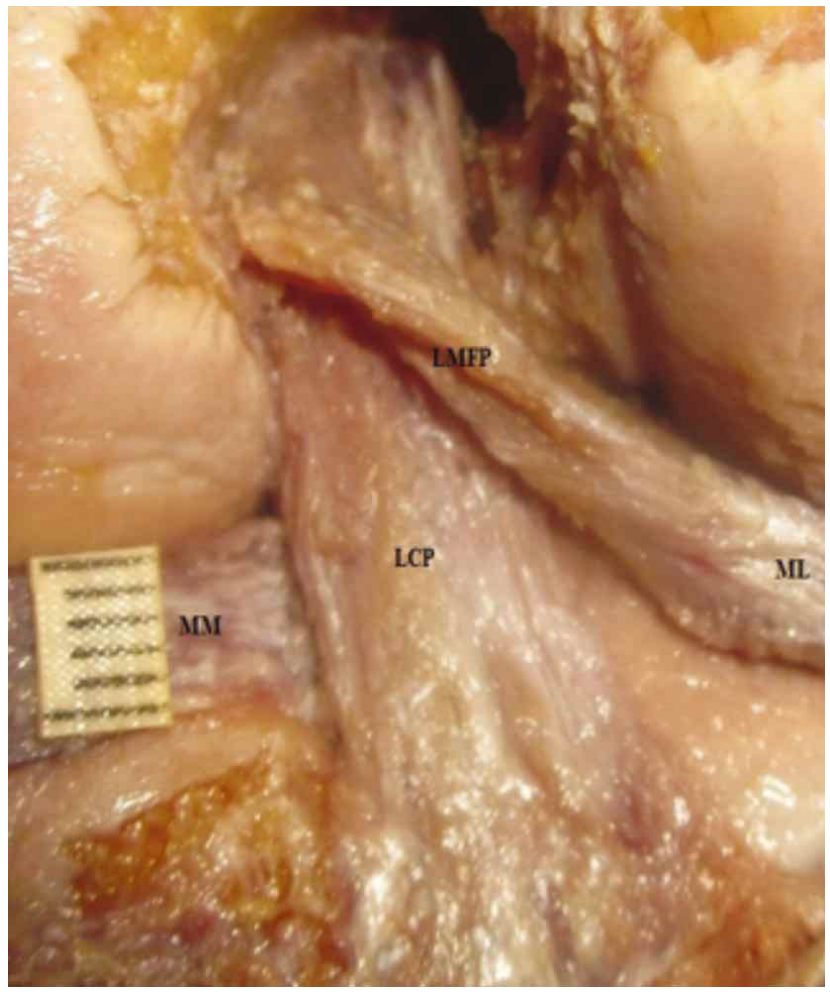

Fig. 10. Vista posterior de la articulación de rodilla derecha. LMFP. Ligamento meniscofemoral posterior; LCP. Ligamento cruzado posterior; ML. Menisco lateral; MM. Menisco medial. El LMFP tiene una trayectoria tipo I relación tipo C. 


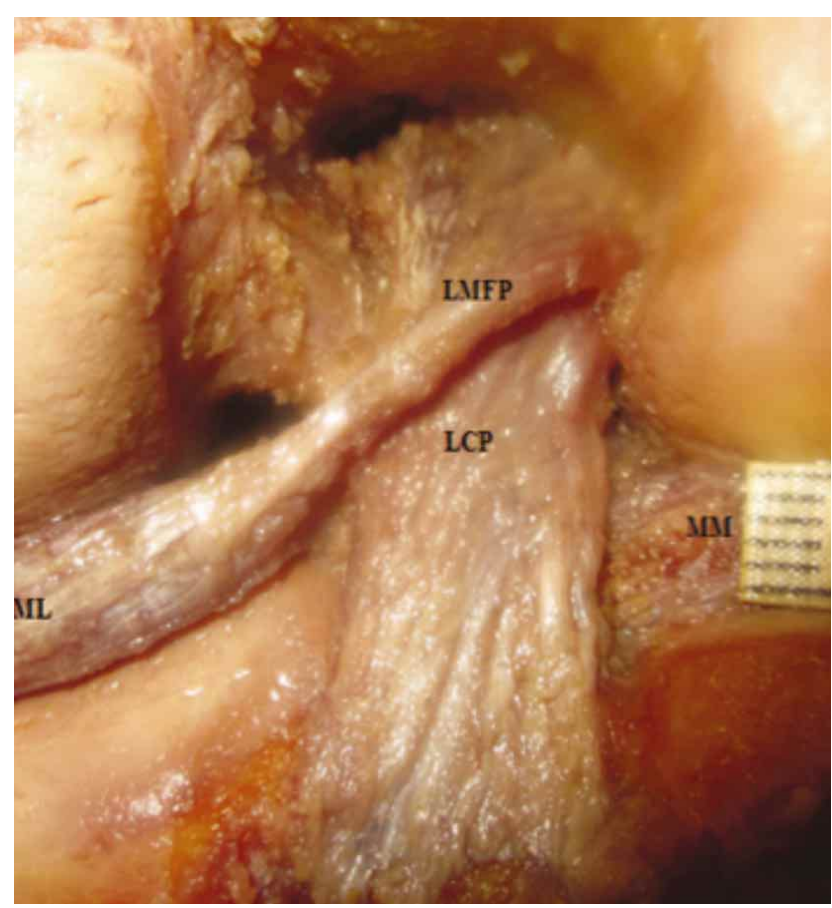

Fig. 11. Vista posterior de la articulación de la rodilla derecha. LMFP. Ligamento meniscofemoral posterior; LCP. Ligamento cruzado posterior; MM. Menisco medial; ML. Menisco lateral. El LMFP presenta una trayectoria tipo II relación B.

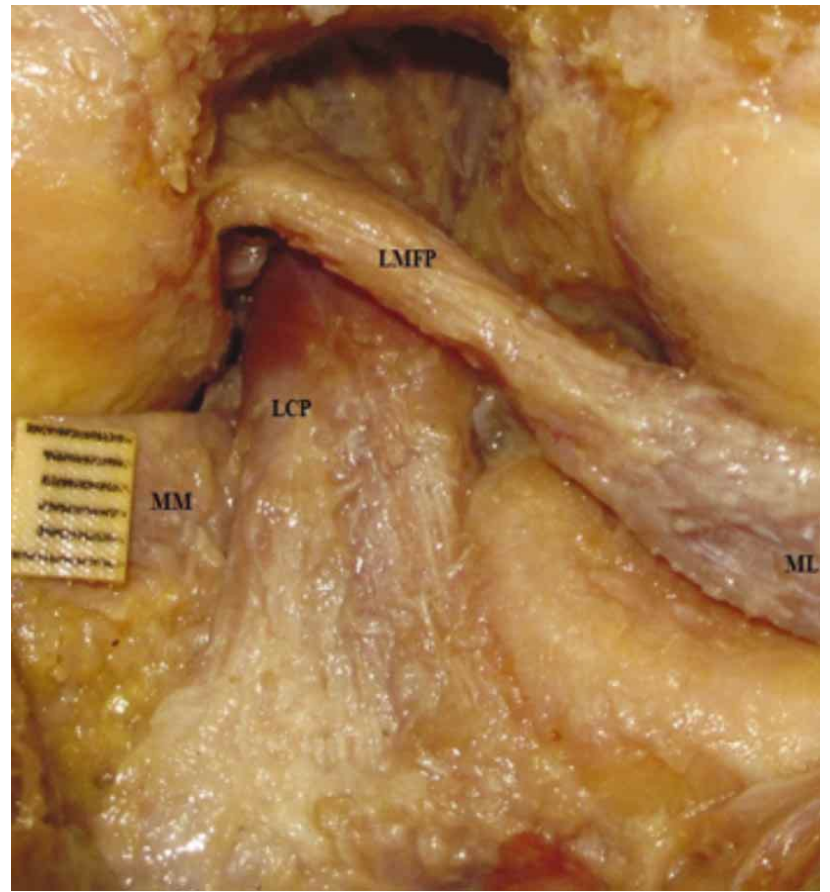

Fig. 12. Vista posterior de la articulación de la rodilla izquierda. LMFP. Ligamento meniscofemoral posterior; LCP. Ligamento cruzado posterior; MM. Menisco medial; ML. Menisco lateral. El LMFP tiene una trayectoria tipo II relación C.

Tabla II. Clasificación del ligamento meniscofemoral posterior.

\begin{tabular}{lcccccccc}
\hline Tipos de & \multicolumn{4}{c}{ Sub clasificación } & \multicolumn{3}{c}{ Total } \\
LMFP & & A & \multicolumn{1}{c}{ B } & C & & & \\
& $\mathbf{n}$ & $\mathbf{\%}$ & $\mathbf{n}$ & $\mathbf{\%}$ & $\mathbf{n}$ & $\mathbf{\%}$ & $\mathbf{n}$ & $\mathbf{\%}$ \\
\hline I & 4 & 19,1 & 8 & 38,1 & 4 & 19,1 & 16 & 76,2 \\
II & 0 & 0 & 2 & 9,5 & 3 & 14,3 & 5 & 23,8 \\
Total & 4 & 19,1 & & & & 33,3 & 21 & 100 \\
\hline
\end{tabular}

\section{DISCUSIÓN}

En general, el trayecto de los LMF no se describe con detalle en la literatura y no existe una clasificación de éstos. Diversos autores describieron una mayor presencia del LMFP sobre el LMFA, entre ellos, Candiolo \& Gautero, LMFP $60 \%$ - LMFA $50 \%$; Kusuyama et al., LMFP $77 \%$ LMFA $69 \%$; Wan \& Felle, LMFP $93 \%$ - LMFA $33 \%$. Por su parte, Poynton et al., describieron un $90 \%$ para el LMFP y un $33 \%$ para el LMFA; Gupte et al. (2002) un $68 \%$ para el LMFP y un $64 \%$ para el LMFA, mientras que Villarroel \& Olave (2016) informaron un $72 \%$ para el LMFP y un 28 $\%$ para el LMFA.
Los LMF fueron categorizados a través de RM por Cho et al., según su inserción proximal, en tipo I (inserción en el cóndilo femoral medial), tipo II (inserción en la mitad proximal del LCP) y tipo III (inserción en la mitad distal del LCP) e inserción distal vertical u oblicua. Del LMFP se encontraron 41 rodillas tipo I, de éstas 31 ligamentos con inserción distal oblicua, 4 con vertical y en 6 no determinada; 28 de tipo II, la inserción oblicua se encontró en 20 casos, en 3 vertical y en 5 casos no clasificaron; el tipo III en 19 muestras, 13 con inserción oblicua, 3 con vertical y 3 no determinada; 2 rodillas no clasificaron dentro de los tipos 
de inserción proximal, presentando una inserción distal tipo oblicuo.

Del LMFA sólo se hallaron 6 de tipo I, 2 con inserción distal oblicua y 4 no determinada; en 11 casos no fue posible clasificar el tipo de inserción proximal, de éstos 2 presentaron inserción distal oblicua y en 9 casos no se ajustaron con los tipos.

La clasificación del LMFP de estos autores no concuerda a lo observado en esta investigación, debido a que el LMFP siempre presentó inserción en el cóndilo femoral medial aunque se uniera en su recorrido al LCP. Con respecto al LMFA, sólo se encontró en 13 casos por lo que no se pudo realizar una clasificación que pudiera ser comparada con estos autores, además que su trayectoria no presentó variaciones en su disposición y todos se insertaron en el cóndilo femoral medial.

Han et al. (2012) propusieron una clasificación para las rodillas en dos categorías, la tipo I estaba sólo el LCP y en la tipo II estaba presente también el LMFP. Describieron una sub clasificación: Ia (8\%) sin fibras oblicuas en el LCP y Ib $(5 \%)$ en el caso de existir un haz de fibras oblicuas en la superficie anterior o posterior del LCP sin insertarse en el menisco lateral. El tipo II, fue dividido en IIa (51\%) si el punto de cruce del LMFP sobre el LCP era mayor al $50 \%$ de la longitud del LCP, en IIb (11\%) si el punto de cruce es menor al $50 \%$ del recorrido del LCP, IIc (24\%) cuando fibras del LMFP se unen al LCP y IId (1\%) fibras del LMFP no se unen al LCP, pero este último presenta un haz de fibras oblicuas en su recorrido. De esta clasificación consideramos las características del tipo II subtipo c y d, en este estudio la mayoría de las fibras se unen en su recorrido al LCP $(66,6 \%)$ y un menor porcentaje no se une éste $(33,3$ $\%$ ), por lo tanto al comparar sólo estos dos subtipos existe concordancia en que la mayoría de los casos el LMFP se une al LCP.

Los hallazgos en este estudio sólo nos permitieron formular una clasificación para el LMFP. El tipo I corresponde al ligamento que nace del menisco lateral y se extiende posterior al LCP en dirección superior y medial hasta la cara lateral del cóndilo medial del fémur y el tipo II se origina del menisco lateral, luego se produce un giro de las fibras, las que se encontraban laterales rotan en dirección anteromedial y las fibras mediales se dirigen hacia posterior y lateral. Cerca de la zona de inserción, las fibras vuelven a su disposición original para insertarse en la cara lateral del cóndilo medial del fémur. Dentro de estos dos tipos se propone una categorización dependiendo de la adherencia de las fibras del LMFP al LCP. Si las fibras del LMFP se unen con el LCP cuando entra en contacto con éste, corresponde al subtipo A, el subtipo B cuando las fibras del LMFP se unen en el tercio final de su trayectoria al LCP y el subtipo C donde no existe unión de las fibras del LMFP con el LCP. En este estudio el tipo I se presentó en el 76,2\% de los casos y dentro de éstos el A corresponde al 19,1\%, el B en el $38,1 \%$ y el C $19 \%$ de las muestras. Con respecto al tipo II se visualizó en el $23,8 \%$ de los casos, el subtipo B se halló en el 9,5\%, el C en el 14,3\% y no se encontró el subtipo A.

Debido a la disposición de los LMF y su relación con el LCP, se realizó análisis de correlación. Se determinó una correlación positiva moderada entre la distancia ELFML y la longitud del LCP, estadísticamente significativa (valor $\mathrm{p}=0,02$ ) y de esta misma distancia con la longitud del LMFP, siendo estadísticamente significativa (valor $\mathrm{p}=$ 0,003). Pudiendo atribuirse que la longitud del LCP corresponde en un $18 \%$ de los casos a la distancia ELF-ML y la longitud del LMFP en un $37 \%$ de los casos a este parámetro. No hay correlación entre este parámetro y la longitud del LMFA (valor $\mathrm{p}=0,81$; no estadísticamente significativo). Por otra parte, se asoció la longitud del LMFP con la longitud del LCP, estableciéndose una correlación positiva moderada, que es estadísticamente significativa (valor $\mathrm{p}=0,03$ ), donde en un $22 \%$ de los casos la longitud del LMFP se relaciona a la longitud del LCP. Con respecto a la longitud del LMFA y del LCP, se observó una correlación positiva baja, no estadísticamente significativa (valor $\mathrm{p}=0,42$ ). En relación a los anchos se determinó que no existe correlación entre el ancho medio del LMFP y del LCP (valor $\mathrm{p}=0,81$; no estadísticamente significativo), sin embargo existe una correlación negativa baja entre el ancho medio del LMFA y el ancho medio del LCP, no estadísticamente significativa (valor $\mathrm{p}=0,51$ ). En la revisión bibliográfica no se hallaron reportes sobre éstas correlaciones, de tal forma no se pudieron confrontar nuestros valores con otras investigaciones.

Los parámetros estudiados complementarán el conocimiento de estos ligamentos, aportando datos morfológicos y biométricos a la anatomía clínica y quirúrgica de la rodilla.

VILLARROEL, G. \& OLAVE, E. Anatomical relationships and statistical correlations between the meniscofemoral ligaments and the posterior cruciate ligament in Chilean individuals. Int. J. Morphol., 34(4):1259-1265, 2016.

SUMMARY: Among the structures that make up the knee joint, the meniscofemoral ligaments (MFL) are considered unstable elements which are related to the posterior cruciate ligament (PCL). In order to correlate the MFL with the PCL and classify the posterior meniscofemoral (PMFL) with respect to PCL, 30 fixed knees of Chilean individuals were studied, all belonging to the Anatomy 
Laboratory of the Faculty of Medicine of the Universidad Catolica del Maule, Chile. The measures were recorded with an electronic caliper and samples were photographed. Regarding course of fibers of the PMFL, $76 \%$ of the cases had a straight direction (type I) and $24 \%$ had a rotation on its axis (type II). In addition, the fibers of PMFL were fused with PCL $(66.7 \%)$ and a lower percentage reached directly and independently to the medial femoral condyle. Averages were obtained and correlated. Thus, it was determined that there is correlation between the lateral epicondyle of the femurlateral malleolus distance (LEF-LM) and the length of the PCL, and this same distance with de PMFL length. Also there was correlation between the length of the PCL and the length of PMFL.

KEY WORDS: Anatomy; Knee; Meniscofemoral ligaments; Posterior cruciate ligament.

\section{REFERENCIAS BIBLIOGRÁFICAS}

Brantigan, O. C. \& Voshell, A. F. Ligaments of the knee joint; the relationship of the ligament of Humphry to the ligament of Wrisberg. J. Bone Joint Surg. Am., 28:66, 1946.

Candiolo, L. \& Gautero, G. Morphologie et fonction des ligaments ménisco-fémoraux de l'articulation du genou chez l'homme. Acta Anat., 38:304-23, 1959.

Cho, J. M.; Suh, J. S.; Na, J. B.; Cho, J. H.; Kim, Y.; Yoo, W. K.; Lee, H. Y. \& Chung, I. H. Variations in meniscofemoral ligaments at anatomical study and MR imaging. Skeletal Radiol., 28(4):189-95, 1999.

Gupte, C. M.; Smith, A.; Jamieson, N.; Bull, A. M.; Thomas, R. D. \& Amis, A. A. Meniscofemoral ligaments--structural and material properties. J. Biomech., 35(12):1623-9, 2002.

Gupte, C. M.; Bull, A. M.; Thomas, R. D. \& Amis, A. A. A review of the function and biomechanics of the meniscofemoral ligaments. Arthroscopy, 19(2):161-71, 2003.

Han, S. H.; Kim, D. I.; Choi, S. G.; Lee, J. H. \& Kim, Y. S. The posterior meniscofemoral ligament: morphologic study and anatomic classification. Clin. Anat., 25(5):634-40, 2012.

Harner, C. D.; Livesay, G. A.; Kashiwaguchi, S.; Fujie, H.; Choi, N. Y. \& Woo, S. L. Comparative study of the size and shape of human anterior and posterior cruciate ligaments. J. Orthop. Res., 13(3):429-34, 1995.

Kusayama, T.; Harner, C. D.; Carlin, G. J.; Xerogeanes, J. W. \& Smith, B. A. Anatomical and biomechanical characteristics of human meniscofemoral ligaments. Knee Surg. Sports Traumatol. Arthrosc., 2(4):234-7, 1994.

Poynton, A. R.; Javadpour, S. M.; Finegan, P. J. \& O`Brien, M. The meniscofemoral ligaments of the knee. J. Bone Joint Surg. Br., 79(2):327-30, 1997.
Radoïévitch, S. Les ligaments des ménisques interarticulaires du genou. Ann. Anat. Path., 8:400-8, 1931.

Rouvière, H. \& Delmas, A. Anatomía Humana Descriptiva, Topográfica y Funcional. Tomo 3. Miembros. 11ª ed., Barcelona, Masson, 2005.

Testut, L. \& Jacob, O. Tratado de Anatomía Topográfica. $8^{\mathrm{a}}$ ed. Barcelona, Salvat, 1961.

Villarroel, G. \& Olave, E. Características biométricas de los ligamentos meniscofemorales en individuos chilenos. Int. J. Morphol., 34(3):860-5, 2016.

Wan, A. C. \& Felle, P. The menisco-femoral ligaments. Clin. Anat., 8(5):323-6, 1995.

\author{
Dirección para Correspondencia: \\ Dr. Enrique Olave \\ Facultad de Medicina \\ Universidad de La Frontera \\ Temuco \\ CHILE
}

Email: enrique.olave@ufrontera.cl

Recibido : 20-08-2016

Aceptado: 28-09-2016 